06

\title{
Кинетические особенности образования зародышей новой фазы в электрическом поле в ряде свинцово-содержащих релаксоров
}

\author{
(C) Л.С. Камзина, Л.А. Кулакова \\ Физико-технический институт им. А.Ф. Иофрфе РАН, \\ Санкт-Петербург, Россия \\ E-mail: kamzin@mail.ioffe.ru
}

(Поступила в Редакцию 12 апреля 2017 г.)

Исследована кинетика и динамика образования зародышей новой фазы во внешнем электрическом поле $E \|$ [001] в сегнетоэлектрических релаксорах $\mathrm{PbIn}_{1 / 2} \mathrm{Nb}_{1 / 2} \mathrm{O}_{3}-\mathrm{PbMg}_{1 / 3} \mathrm{Nb}_{2 / 3} \mathrm{O}_{3-x} \mathrm{PbTiO}_{3}(x=25$ и $32 \%)$ и $\mathrm{PbMg}_{1 / 3} \mathrm{Nb}_{2 / 3} \mathrm{O}_{3}-29 \mathrm{PbTiO}_{3}$. При комнатной температуре проведены совместные измерения оптического пропускания на разных длинах волн, а также скорости и затухания звука на разных частотах. Проведен анализ характера изменений оптических и акустических свойств в различных электрических полях с целью выявления механизмов таких изменений в условиях фазового перехода. Уменьшение оптического пропускания со временем связано только с изменением размеров нанообластей в процессе фазового перехода. Наблюдаемые акустические аномалии являются следствием фазового перехода и обусловлены увеличением жесткости решетки, вызванной большим пьезоэлектрическим эффектом в сегнетоэлектрической фазе.

DOI: $10.21883 /$ FTT.2017.10.44963.129

\section{1. Введение}

Релаксорные сегнетоэлектрики - это особый класс материалов, которые легко поляризуются внешним электрическим полем и обладают уникальными электромеханическими свойствами. Эти свойства делают релаксоры привлекательными материалами для применения в качестве сенсоров и актюаторов. Присутствие локальных кластеров со случайно ориентированной поляризацией, известных как полярные нанообласти (PNR), играет ключевую роль в понимании необычных свойств релаксоров. Общей чертой всех релаксорных твердых растворов, таких как $\mathrm{PbMg}_{1 / 3} \mathrm{Nb}_{2 / 3} \mathrm{O}_{3-x} \mathrm{PbTiO}_{3}$ (PMN-xPT), $\mathrm{PbIn}_{1 / 2} \mathrm{Nb}_{1 / 2} \mathrm{O}_{3}-\mathrm{PbMg}_{1 / 3} \mathrm{Nb}_{2 / 3} \mathrm{O}_{3}-x \mathrm{PbTiO}_{3} \quad(\mathrm{PIN}-\mathrm{PMN}$ $-x \mathrm{PT}), \mathrm{PbZn}_{1 / 3} \mathrm{Nb}_{2 / 3} \mathrm{O}_{3}-x \mathrm{PbTiO}_{3} \quad(\mathrm{PZN}-x \mathrm{PT})$ и ряда других, является случайное расположение ионов в кристаллографически эквивалентных положениях кристаллической решетки. Случайно ориентированные локальные электрические и упругие поля служат причиной возникновения PNR, которые являются предшественниками сегнетоэлектрического фазового перехода. При высоких температурах, на несколько сотен градусов выше температуры максимума диэлектрической проницаемости, релаксоры существуют в неполярной параэлектрической кубической фазе, которая подобна по многим параметрам параэлектрической фазе нормальных сегнетоэлектриков. При охлаждении релаксоры трансформируются в эргодическое релаксорное состояние, в котором при температуре Бернса $\left(T_{\mathrm{B}} \sim 620-650 \mathrm{~K}\right)$ появляются PNR c хаотически распределенными направлениями дипольных моментов. Присутствие PNR проявлялось в ряде физических явлений, таких как отклонение температурных зависимостей величины двупреломления и относительного удлинения от линейного закона, характерного для более высоких температур [1-3], акустической эмиссии [4] и др. Эти динамические области, внедренные в кубическую матрицу, характеризуются локальной ромбоэдрической симметрией. Возникшие PNR имеют размеры 2-3 nm и остаются практически неизменными при понижении температуры вплоть до температуры Кюри $\left(T_{\mathrm{C}}\right)$. Вблизи $T_{\mathrm{C}}$ размеры этих областей начинают резко возрастать и достигают $\sim 7 \mathrm{~nm}$. С дальнейшим понижением температуры ниже температуры замерзания размеры их уже $\sim 10-30 \mathrm{~nm}$. При этой температуре возникает неэргодическое стеклоподобное состояние c замороженной локальной поляризацией. Локальная структура PNR обычно отличается от средней макроскопической структуры решетки, в которой они находятся. В замороженном релаксорном состоянии PNR сохраняют слабую кинетику, но дальний порядок не возникает. Свойства кристалла в таком неравновесном состоянии должны зависеть от времени. Это было подтверждено в работах по изучению диэлектрических свойств [5-7], а также оптическими и акустическими исследованиями [8-10]. В работе [10] мы изучали кинетику прохождения сегнетоэлектрического фазового перехода только в релаксорах $\mathrm{PIN}-\mathrm{PMN}-x \mathrm{PT}$, используя оптические и акустические измерения. Именно совместное наблюдение изменений во времени оптических и акустических свойств в электрическом поле позволяет проследить особенности динамики формирования полярных областей в процессе фазового перехода из стеклоподобной в сегнетоэлектрическую фазу. Нами было высказано предположение, что изменение оптических свойств связано главным образом с изменением размеров неоднородностей. При приложении электрического поля в стеклоподобной фазе с течением времени количество областей с локальной поляризацией возрастает. Увеличиваются и их размеры. Как только размеры становятся сравнимыми с длиной волны света $(\lambda=0.63 \mu \mathrm{m})$, происходит умень- 
шение оптического пропускания вследствие рассеяния света. Было показано [10], что в электрическом поле, приложенном вдоль кристаллографической оси [001], резкое изменение акустических свойств (скорости звука и затухания) происходит с заметной задержкой по времени относительно изменения оптического пропускания. В этой связи возникает вопрос, какой механизм определяет акустические аномалии? По аналогии можно предположить, что, как и в оптике, процесс определяется размерами полярных областей, поскольку длина ультразвуковой волны $\left(\Lambda_{S}\right)$ существенно больше длины волны света $\left(\Lambda_{S} \gg \lambda\right)$. Однако такие предположения нуждаются в дополнительных исследованиях.

В настоящей работе проведены одновременные измерения изменения оптических (для двух длин волн) и акустических (для двух частот, т.е. двух длин акустических волн) свойств не только в тройных (PIN-PMN-xPT), но и в двойных $(\mathrm{PMN}-x \mathrm{PT})$ твердых растворах с целью получения более полной информации о механизмах, определяющих такие изменения.

\section{2. Образцы и методика эксперимента}

Кристаллы выращивали прямо из расплава модифицированным методом Бриджмена [11]. Были получены высококачественные монокристаллы 20PIN55PMN-25PT (PT25), 33PIN-35PMN-32PT (РT32) и PMN-29PT. Как следует из рентгеновских и нейтронных исследований, симметрия всех исследуемых образцов при комнатной температуре в отсутствие электрического поля в целом кубическая. Эта симметрия является усредненной и играет роль матрицы, в которую внедрены нанообласти разной локальной симметрии. В кристаллах РТ25 это неупорядоченные хаотически расположенные области ромбоэдрической фазы. В кристаллах PMN-29PT и PT32, имеющих ромбоэдрическое искажение, это отдельные области моноклинной фазы, а в РТ32 - еще и тетрагональной фазы [8]. Кристаллы ориентировались и затем разрезались вдоль плоскостей (001). Для оптических измерений использовались $\mathrm{He}-\mathrm{Ne}$-лазер $(\lambda=0.63 \mu \mathrm{m}$, непрерывный режим $)$ и полупроводниковый лазер $(\lambda=1.3 \mu \mathrm{m}$, импульсный режим). Измерения проводили при комнатной температуре. Электрическое поле прикладывалось в направлении [001] в стеклоподобной релаксорной фазе и совпадало по направлению с распространением звуковой волны. Пропускание света измеряли в перпендикулярном [100] направлении. При непрерывном режиме работы лазера $(\lambda=0.63 \mu \mathrm{m})$ импульс света формировался дисковым прерывателем. Им же вырабатывался импульс синхронизации запуска ВЧ генератора, вырабатывающего акустический импульс. В инфракрасном диапазоне $(\lambda=1.3 \mu \mathrm{m})$ синхронизация оптических и акустических импульсов осуществлялась сигналом ВЧ генератора. Для измерения акустических свойств кристаллов использовали эхо-импульсную методику [4]. Ультразвуковые импульсы частотой 10 и $20 \mathrm{MHz}$ и длительностью до $0.5 \mu$ s возбуждались с помощью генератора сигналов AFG фирмы INSTEK резонансными пластинками из пьезоэлектрической керамики, приклеиваемыми к образцу. Широкополосный (полоса $200 \mathrm{MHz}$ ) двухлучевой осциллограф (фирмы Tectronix) и камера Sony Cybershot использовались для фиксированной и непрерывной записи поведения оптических и акустических импульсов. Перед каждым измерением образцы отжигали в течение часа при $\sim 500 \mathrm{~K}$. После отжига образцы охлаждали без поля до комнатной температуры, при которой к ним прикладывалось постоянное электрическое поле с напряженностью, достаточной для индуцирования фазового перехода в сегнетоэлектрическое состояние. После приложения поля осуществляли регистрацию изменения оптического пропускания, положения и амплитуды акустических импульсов со временем. Измерения проводили спустя некоторое время $t_{r}$ после отжига кристалла.

\section{3. Экспериментальные результаты}

На рис. 1 представлена динамика изменения затухания (кривые 1,2) и скорости звука (кривые 3,4) на двух частотах $F=10$ и $20 \mathrm{MHz}\left(\Lambda_{S} \sim 230-450 \mu \mathrm{m}\right)$, а также оптического пропускания $(5,6)(\lambda=0.63 \mu \mathrm{m})$ в электрическом поле для кристаллов РТ25 (два цикла измерений). Для того чтобы избежать различия во влиянии внутренних электрических полей, формирующихся с течением времени в процессе старения релаксорной фазы, измерения проводили при одном и том же времени $\left(t_{r}\right)$, прошедшем между началом измерений и отжигом кристалла. Определенные трудности, существующие при определении этого времени, преодолевались нами одновременным выполнением оптических и акустических измерений. Так как оптическое пропускание сильно зависит от $t_{r}$ [9], то его воспроизведение при циклах измерений с различными акустическими частотами (рис. 1, кривые 5,6) позволяет нам дополнительно контроли-

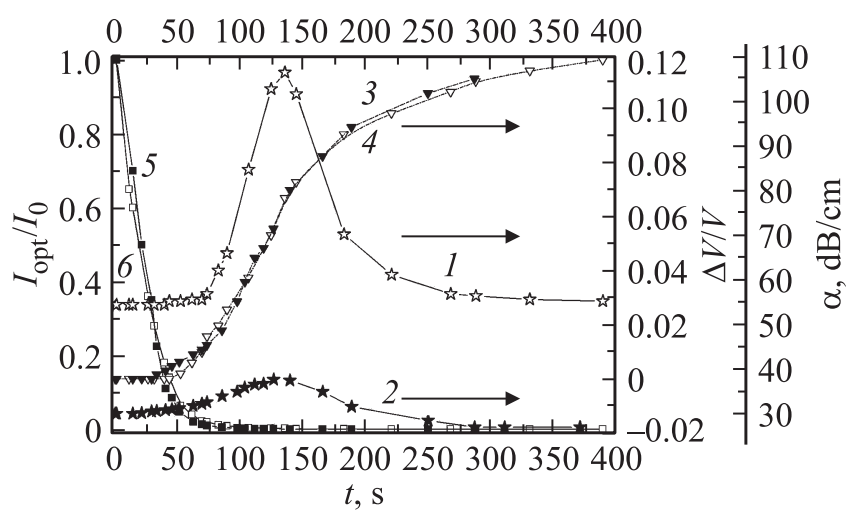

Рис. 1. Изменение со временем затухания $(1,2)$, скорости звука $(3,4)$, оптического пропускания $(5,6)$ в электрическом поле $E=1.5 \mathrm{kV} / \mathrm{cm}$ в кристалле РТ25 при разных частотах звука: темные точки $-10 \mathrm{MHz}$, светлые точки $-20 \mathrm{MHz}$; $\lambda=0.63 \mu \mathrm{m}, t_{r}=1 \mathrm{~h}$. 
ровать одинаковое исходное состояние образцов перед измерениями.

Из приведенных на рис. 1 зависимостей скорости звука и затухания видно, что положение акустических аномалий (резкий рост скорости и максимум затухания) не зависит от частоты, а следовательно, и от длины волны звука. Из этого можно сделать вывод, что аномалии эти - не размерного характера, а возникают вследствие изменения упругих свойств в объеме, характерного для фазового перехода. В области фазового перехода в узком интервале времени эти величины резко меняются. Наблюдается большой пик затухания, что является естественным в условиях перестройки кристаллической структуры, и резкое возрастание скорости звука из-за увеличения жесткости решетки, вызванного большим пьезоэлектрическим эффектом в сегнетоэлектрической фазе. Аналогичные зависимости получены и для кристалла PMN-29PT.

Эти результаты позволяют утверждать, что акустические измерения являются надежным инструментом выявления фазового перехода в исследованном классе материалов.

С целью изучения кинетических особенностей образования зародышей новой фазы во внешнем электрическом поле и механизма, ответственного за уменьшение оптической прозрачности в исследуемых кристаллах, нами были выполнены совместные измерения изменения со временем оптического пропускания на разных длинах волн, а скорости и затухания звука при фиксированной частоте $(F=20 \mathrm{MHz})$. На рис. 2 представлены соответствующие зависимости для кристаллов РТ25 в электрическом поле $E=1.5 \mathrm{kV} / \mathrm{cm}$ на двух длинах волн: два цикла измерений $\lambda=0.63 \mu \mathrm{m}$ (кривая 1 ) и $1.3 \mu \mathrm{m}$ (кривая 2). Зависимости оптического пропускания, а также затухания (кривые 3,4 ) и скорости звука (кривые 5,6 ) на частоте $F=20 \mathrm{MHz}$ снимали спустя одинаковое время после отжига $\left(t_{r}=1 \mathrm{~h}\right)$. Как видно из рис. 2, поведение акустических свойств воспроизводится для обоих циклов измерений (кривые 3-6). Так как положение акустических аномалий сильно зависит от $t_{r}$ [9], то их совпадение при двух циклах измерений с различными длинами волн света позволяет нам дополнительно контролировать одинаковое исходное состояние образцов перед измерениями.

Перед началом измерений (в отсутствие поля), как видно из рис. 2, оптическая прозрачность максимальна на обеих длинах волн. Это неудивительно, так как эти кристаллы лежат достаточно далеко от МФГ, и в отсутствие поля в стеклоподобной релаксорной фазе сосуществуют нанообласти ромбоэдрической и моноклинной фаз, размеры которых меньше длины волны света, как для $0.63 \mu \mathrm{m}$, так и для $1.3 \mu \mathrm{m}$.

В присутствии электрического поля оптическое пропускание в инфракрасной области на длине волны $1.3 \mu \mathrm{m}$ (кривая 2) уменьшается очень медленно, в отличие от его поведения в видимой области на длине $0.63 \mu \mathrm{m}$ (кривая 1). Наиболее заметное уменьшение

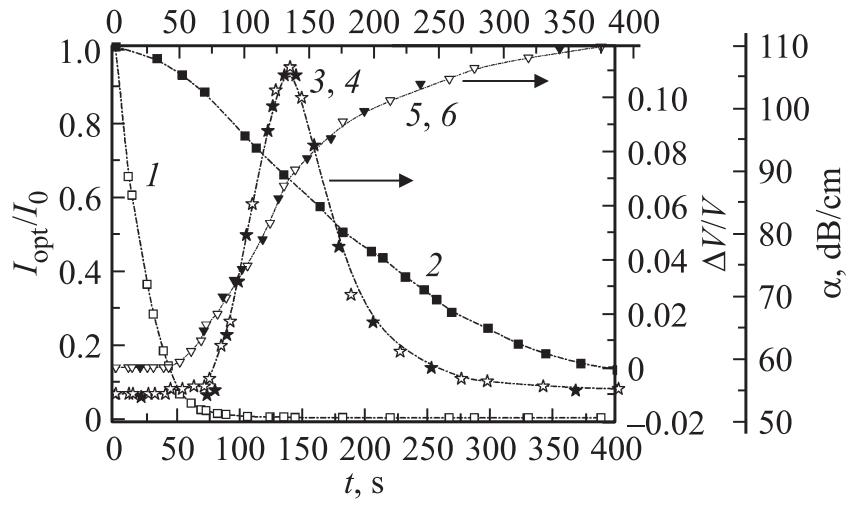

Рис. 2. Временны́е зависимости оптического пропускания $(1,2)$, затухания $(3,4)$ и скорости звука $(5,6)$ в электрическом поле $E=1.5 \mathrm{kV} / \mathrm{cm}$ в кристалле РТ25 при разных длинах волн света: светлые точки - $\lambda=0.63 \mu \mathrm{m}$, темные точки $\lambda=1.3 \mu \mathrm{m} ; F=20 \mathrm{MHz}, t_{r}=1 \mathrm{~h}$.

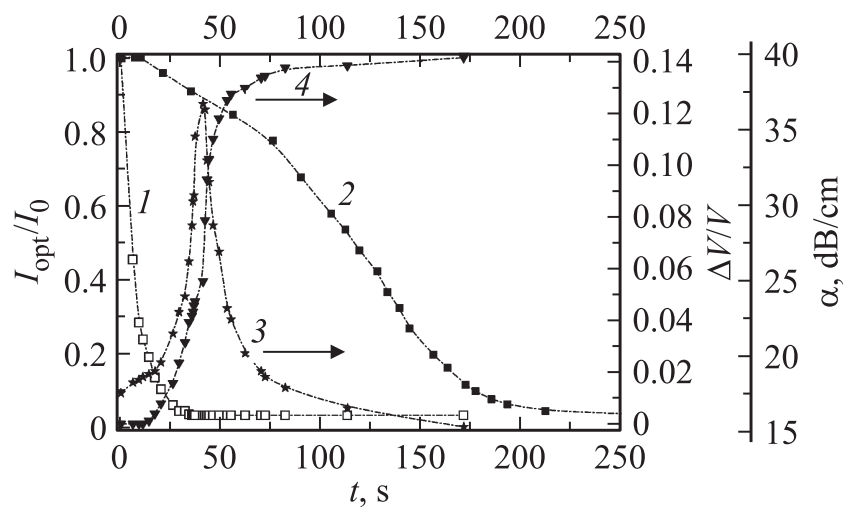

Рис. 3. Кинетика изменений оптического пропускания $(1,2)$, затухания (3) и скорости звука (4) в электрическом поле $E=1.23 \mathrm{kV} / \mathrm{cm}$ в кристалле $\mathrm{PMN}-29 \mathrm{PT}$ при разных длинах волн света, $\lambda, \mu \mathrm{m}: 1-0.63,2-1.3 ; F=20 \mathrm{MHz}, t_{r}=1 \mathrm{~h}$ $20 \mathrm{~min}$.

пропускания на длине волны $1.3 \mu \mathrm{m}$ наблюдается после аномалий акустических свойств (кривые 3-6), которые являются, как мы показали выше, следствием фазового перехода. Аналогичное поведение изменения оптического пропускания в инфракрасной области наблюдается в кристаллах PMN-29PT (рис. 3). Это значит, что в процессе фазового перехода не происходит полной поляризации образца: для поляризации областей размером порядка инфракрасной длины волны $(1.3 \mu \mathrm{m})$ и более требуется больше времени. Вкладом именно этих областей определяется медленное увеличение скорости звука после перехода во всех исследованных образцах (рис. 1-3).

Интересные результаты получены нами в кристаллах PIN-PMN-32PT, лежащих ближе к МФГ. Так же, как и в представленных выше кристаллах, соответствующие аномалии акустических свойств свидетельствуют о фазовом переходе в электрическом поле (рис. 4, кривые 1,2). Изменения оптических свойств в этих кристаллах на 


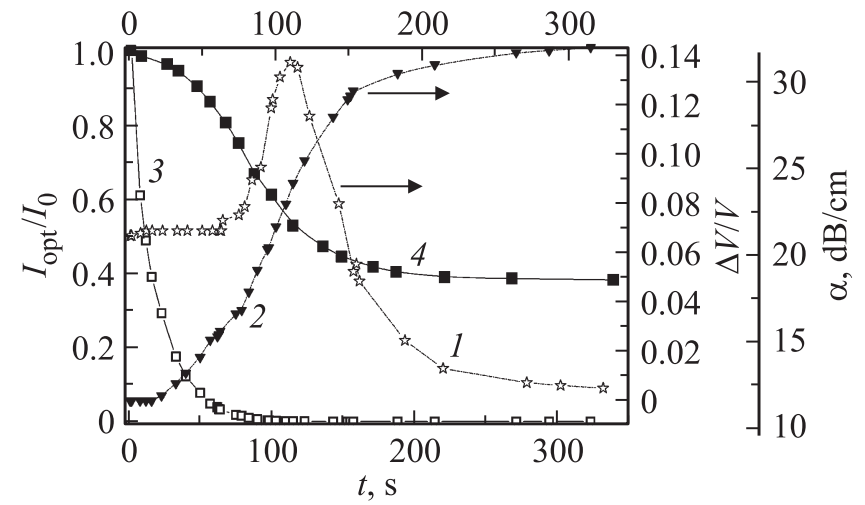

Рис. 4. Изменение со временем затухания (1), скорости звука (2) и оптического пропускания $(3,4)$ в электрическом поле $E=2.1 \mathrm{kV} / \mathrm{cm}$ в кристалле РТ32 при разных длинах волн света, $\lambda, \mu \mathrm{m}: 3-0.63,4-1.3 ; F=20 \mathrm{MHz}, t_{r}=1 \mathrm{~h}$.

разных длинах волн существенно различаются. На рис. 4 (кривые 3,4) представлены зависимости пропускания в электрическом поле, приложенном вдоль [001]. В этих образцах в отсутствие поля кроме нанообластей ромбоэдрической и моноклинной симметрии существуют нанообласти тетрагональной фазы [8]. Так же как и в кристаллах PT25 и PMN-29PT, пропускание света в отсутствие поля максимально, что свидетельствует о малости размеров всех сосуществующих областей. После перехода в электрическом поле в моноклинную фазу [12,13] оптическое пропускание на длине волны $0.63 \mu \mathrm{m}$ падает на 3-4 порядка (кривая 3), в то время как на длине волны $1.3 \mu \mathrm{m}$ оно уменьшается всего на $60 \%$ (кривая 4). Это может свидетельствовать, что часть сосуществующих областей в данном приложенном поле имеет размеры больше $0.63 \mu \mathrm{m}$, но меньше, чем $1.3 \mu \mathrm{m}$. Скорее всего, это полярные области тетрагональной фазы, для увеличения размеров которых требуются бо́льшие электрические поля.

\section{4. Заключение}

Таким образом, в данной работе впервые проведены одновременные измерения оптических (на двух длинах волн) и акустических свойств (на двух частотах) в тройных (PIN-PMN-xPT, $x=25,32 \%)$ и двойных PMN-29PT твердых растворах с целью получения информации о кинетике образования зародышей новой фазы в электрическом поле.

Обнаружено аномальное изменение во времени акустических свойств (пик поглощения и резкое возрастание скорости звука) в электрическом поле. Наблюдаемые аномалии акустических свойств являются следствием изменения упругих свойств в объеме кристалла, характерного для фазового перехода.

Во всех исследованных кристаллах наблюдается резкое уменьшение оптического пропускания в видимой области спектра с существенным опережением прояв- ления аномалий акустических свойств, в то время как в инфракрасном диапазоне оно происходит в более широком временно́м интервале и наблюдается даже после аномалий акустических параметров. Зависимость пропускания от длины волны света связана только с увеличением размеров нанообластей в процессе прохождения фазового перехода. Высказано предположение, что используемые в работе электрические поля приводят не к полной, а только к частичной поляризации образца, и дальний порядок возникает в части объема кристалла.

Таким образом, измерения оптического пропускания на разных длинах волн позволяют оценить динамику изменения размеров полярных областей в процессе фазового перехода, а акустические измерения являются надежным инструментом выявления фазового перехода в исследованном классе материалов.

\section{Список литературы}

[1] I.W. Chen. J. Phys. Chem. Solids 61, 197 (2000).

[2] G. Burns, F.H. Dacol. Solid State Commun. 48, 853 (1983).

[3] H. Arndt, F. Schmidt. Ferroelectrics 79, 149 (1988).

[4] M. Roth, E. Mojaev, E. Dul'kin, P. Gemeiner, B. Dkhil. Phys. Rev. Lett. 98, 265701 (2007).

[5] E.V. Colla, M.B. Weissman. Phys. Rev. B 72, 104106 (2005).

[6] E.V. Colla, D. Vigil, J. Timmerwilke, M.B. Weissman. Phys. Rev. B 75, 214201 (2007).

[7] E.V. Colla, N. Jurik, Y. Liu, M.E.X. Delgado, M.B. Weissman, D.D. Vieland, Z.-G. Ye. J. Appl. Phys. 113, 184104 (2013).

[8] Л.С. Камзина, Л.А. Кулакова, Н. Luo. ФТТ 56, 9, 1809 (2014).

[9] Л.С. Камзина, Л.А. Кулакова. ФТТ 58, 1, 177 (2016).

[10] Л.С. Камзина, Л.А. Кулакова. ФТТ 59, 2, 290 (2017).

[11] H. Luo, G. Xu, H. Xu, P. Wagn. Jpn. J. Appl. Phys. 39, 5581 (2000).

[12] S.J. Zhang, L. Lebrun, S. Rhee, C.A. Randall, T.R. Shrout. Appl. Phys. Lett. 81, 892 (2002).

[13] M. Shanthi, L.C. Lim. J. Appl. Phys. 106, 114116 (2009). 